\title{
LOS APOYOS ESTUDIANTILES AL SERVICIO DE LA FORMACIÓN INTEGRAL EN LA UNIVERSIDAD DE CHILE
}

\author{
Marcia Oyarce* \\ Iván J. Páez**
}




\section{RESUMEN}

En el presente artículo se describen brevemente tres áreas de acción, que tienen por objetivo apoyar el desarrollo integral de los estudiantes de la Universidad de Chile. El desafío es alcanzar una población de estudiantes de excelencia académica, sin discriminaciones socioeconómicas.

Una de estas áreas es el Bienestar Estudiantil, unidad encargada de la elaboración de programas dirigidos a apoyar a los estudiantes que presentan carencia de recursos económicos y a brindar atención psicosocial. Un segundo ámbito es el Servicio de Atención Médico-Dental, orientado a brindar un modelo de desarrollo de salud integral. Una tercera área es el apoyo a las actividades extracurriculares, que pretende contribuir a la promoción de acciones que busquen el mejoramiento de los proyectos estudiantiles, entregando una asesoría directa a los alumnos.

\section{ABSTRACT}

The article briefly describes three areas of action intending to aid the integral development of students at the Universidad de Chile. The challenge is to end up with a population of students noted for academic excellence, without any socio-economic discrimination.

One of these areas is Student Welfare, which is the unit responsible for preparing programs to assist students who lack the necessary financial resources and provide them with psychosocial care. The second area is the Medical and Dental Health Care Service, which aims to provide integral healthcare. The third area consists of support for extracurricular activities which intends to help promote actions that seek to improve students' projects by providing direct advisory services to the students. 


\section{LOS APOYOS ESTUDIANTILES AL SERVICIO DE LA FORMACIÓN INTEGRAL EN LA UNIVERSIDAD DE CHILE}

\section{PRESENTACIÓN}

La misión de la Universidad de Chile es la generación, cultivo, transmisión e integración de las ciencias, las humanidades, las artes, las técnicas y las demás manifestaciones de la cultura. Dicha misión se realiza a través de una acción prioritariamente orientada hacia los problemas y necesidades nacionales, con el propósito de contribuir al desarrollo integral del país y a la formación de una ciudadanía preparada, crítica, con conciencia social y responsabilidad ética. La Universidad de Chile asume esta misión buscando la excelencia, de modo reflexivo y crítico, a través de sus funciones fundamentales de docencia, investigación, creación artística y extensión.

En la Universidad de Chile, más allá de buscar un título profesional, los estudiantes complementan su formación compartiendo los valores expresados en la vivencia solidaria, en la excelencia académica, y en el respeto y la tolerancia de todas las expresiones ciudadanas que hacen de esta casa de estudios superiores una institución con marcada identidad, el más alto prestigio y una reconocida tradición nacional e internacional.

\section{BIENESTAR ESTUDIANTIL}

La Universidad de Chile asume su tarea en el contexto de un proyecto de país. Desde esta perspectiva, desarrolla la tarea de trabajar en torno a una población de estudiantes de excelencia y potencial académico, sin discriminación socioeconómica. La diversidad social y cultural de nuestros alumnos nos propone un desafío para potenciar nuestros esfuerzos y relacionarnos de mejor manera con nuestros recursos humanos y materiales destinados a apoyarlos durante su formación. 
En este contexto, Bienestar Estudiantil considera que las necesidades de los alumnos no se reducen sólo al ámbito del financiamiento y el pago de aranceles; se propone considerar una realidad más amplia, relacionada con aspectos sociales, culturales y valóricos, orientándose en definitiva hacia un enfoque integral de acción. Es así como los programas y proyectos relacionados con Bienestar Estudiantil están dirigidos a apoyar a los estudiantes en las áreas que presentan mayor carencia: falta de recursos económicos, apoyo familiar inadecuado o inexistente, y difícil acceso a los servicios de apoyo estatal.

\section{A. ATENCIÓN SOCIAL Y CONSEJERÍA}

La consejería personalizada es un método de intervención psicosocial que se centra en la persona, con sus particularidades e intereses, porque postula que ésta es capaz de progresar y autodeterminarse, ya que posee, en sí misma, una parte de las respuestas a las dificultades que encuentra en las conductas que quiere modificar.

\section{B. ATENCIÓN ECONÓMICA}

Corresponde a la administración de subsidios económicos que buscan atenuar situaciones de déficit económico o desigualdad de condiciones de algunos estudiantes. Los programas de apoyo económico se agrupan en Becas Internas, Becas Externas y Becas Internas de Facultades.

\section{B.1 Programa de Becas Internas Véase cuadro $\mathrm{N}^{\circ} 1$}

\section{B.2 Programa de Becas Externas}

La función de Bienestar Estudiantil, en esta área, es postular al estudiante o bien proceder a la renovación del beneficio, de acuerdo a los parámetros establecidos por el Ministerio de Educación y otros organismos.

Las becas a las cuales pueden optar nuestros estudiantes se detallan en el cuadro $\mathrm{N}^{\circ} 2$ adjunto:

\section{B.3 Programas de Becas Internas de las Facultades}

Las distintas unidades académicas otorgan beneficios a sus estudiantes; estas becas pueden ser de alimentación, dinero o aranceles. 
Cuadro $\mathrm{N}^{\circ} 1$ :

Becas internas administradas por el Bienestar Estudiantil Informe al 31 de agosto de 2002

\begin{tabular}{|l|c|c|}
\hline Descripción & $\begin{array}{c}\text { Tipo de } \\
\text { beneficio }\end{array}$ & $\begin{array}{c}\text { Alumnos } \\
\text { beneficiados }\end{array}$ \\
\hline Beca Universidad de Chile & Arancel & 481 \\
Beca Irma Salas & Materiales & 5 \\
Beca Excelencia Deportiva - completa & Arancel & 10 \\
Beca Excelencia Deportiva - media & Arancel & 20 \\
Programa de Atención Económica (PAE) & Mantención & 6.334 \\
Hogares Universitarios & Estadía & 112 \\
Apoyo Preescolar & Mantención & 124 \\
Beca de Emergencia $(*)$ & Mantención & 126 \\
Franquicias - Médicas $(*)$ & Salud & 886 \\
Franquicias - Dental $(*)$ & Salud & 797 \\
Complemento Beca Juan Gómez Millas & Arancel & 133 \\
\hline
\end{tabular}

* Cantidad corresponde al año 2001

\section{Cuadro $\mathrm{N}^{0}$ 2:}

Becas externas administradas por el Bienestar Estudiantil Informe al 31 de agosto de 2002

\begin{tabular}{|l|c|c|}
\hline \multicolumn{1}{|c|}{ Descripción } & $\begin{array}{c}\text { Tipo de } \\
\text { beneficio }\end{array}$ & $\begin{array}{c}\text { Alumnos } \\
\text { beneficiados }\end{array}$ \\
\hline $\begin{array}{l}\text { Beca Juan Gómez Millas } \\
\text { Programa de Fortalecimiento de la Formación } \\
\text { Inicial Docente (Beca Pedagogía) }\end{array}$ & Arancel & 1.166 \\
\hline $\begin{array}{l}\text { Becas de Mantención para Estudiantes } \\
\text { Destacados que Ingresan a Pedagogía }\end{array}$ & Arancel & 51 \\
\hline Beca Hijos de Profesionales de la Educación & Mantención & 15 \\
\hline $\begin{array}{l}\text { Beca Bicentenario } \\
\text { Beca Indígena }\end{array}$ & Arancel & 248 \\
\hline $\begin{array}{l}\text { Beca Presidente de la República } \\
\text { Beca de Reparación }\end{array}$ & Arancel & 1.946 \\
\hline Beca Programa Adopta un Hermano (*) & Mantención & 42 \\
\hline Beca COPESA & Mantención & 754 \\
Beca NISSAN (Fac. Cs. Económicas y Adm.) & Arancel y Mantención & 14 \\
\hline
\end{tabular}

* Financiamiento complementado con recursos internos. 


\section{C. ÁREA DE INVESTIGACIÓN Y PROYECTOS DE INTERVENCIÓN}

Comprende el desarrollo de estudios que permitan evaluar en forma permanente la entrega de beneficios y las necesidades de los estudiantes de la Universidad. Esta labor debe ser el eje central de la definición de las necesidades de la población estudiantil y, por ende, de los programas de apoyo a desarrollar. Además, persigue generar proyectos que permitan obtener apoyos para cubrir necesidades propias del bienestar estudiantil, así como proyectos de intervención social en temáticas relevantes para la comunidad universitaria.

\section{RED DE PROMOCIÓN DE AUTOCUIDADO}

Implementación de redes locales en las cuales se fomente el desarrollo biopsicosocial de los estudiantes, mediante el trabajo multidisciplinario. El objetivo es generar mecanismos que fomenten el autocuidado del estudiante en áreas tales como: estrés, manejo de conflictos, problemas vocacionales, sexualidad, consumo de drogas y alcohol, entre otros.

\section{ATENCIÓN MÉDICO-DENTAL}

Bienestar Estudiantil enfoca actualmente la salud de los alumnos con base en un modelo de desarrollo de salud integral, con atención especial para quienes no cuentan con previsión ni seguro de salud. Para ello, el Servicio Médico y Dental (SEMDA) ofrece un conjunto de servicios destinados a atender necesidades primarias de salud médica y dental de los estudiantes.

Los equipos de profesionales de la salud realizan atención directa; programas preventivos (campañas de vacunación y otros) a través de los diferentes programas de atención médica y dental; cursos electivos curriculares de "autocuidado en salud y calidad de vida"; talleres de primeros auxilios; cursos de preparación al parto y talleres de nutrición, dietoterapia y medición antropométrica. Estos mismos equipos asumen la supervisión indirecta de los casinos de las distintas unidades académicas, así como la supervisión directa en los Hogares Universitarios. 


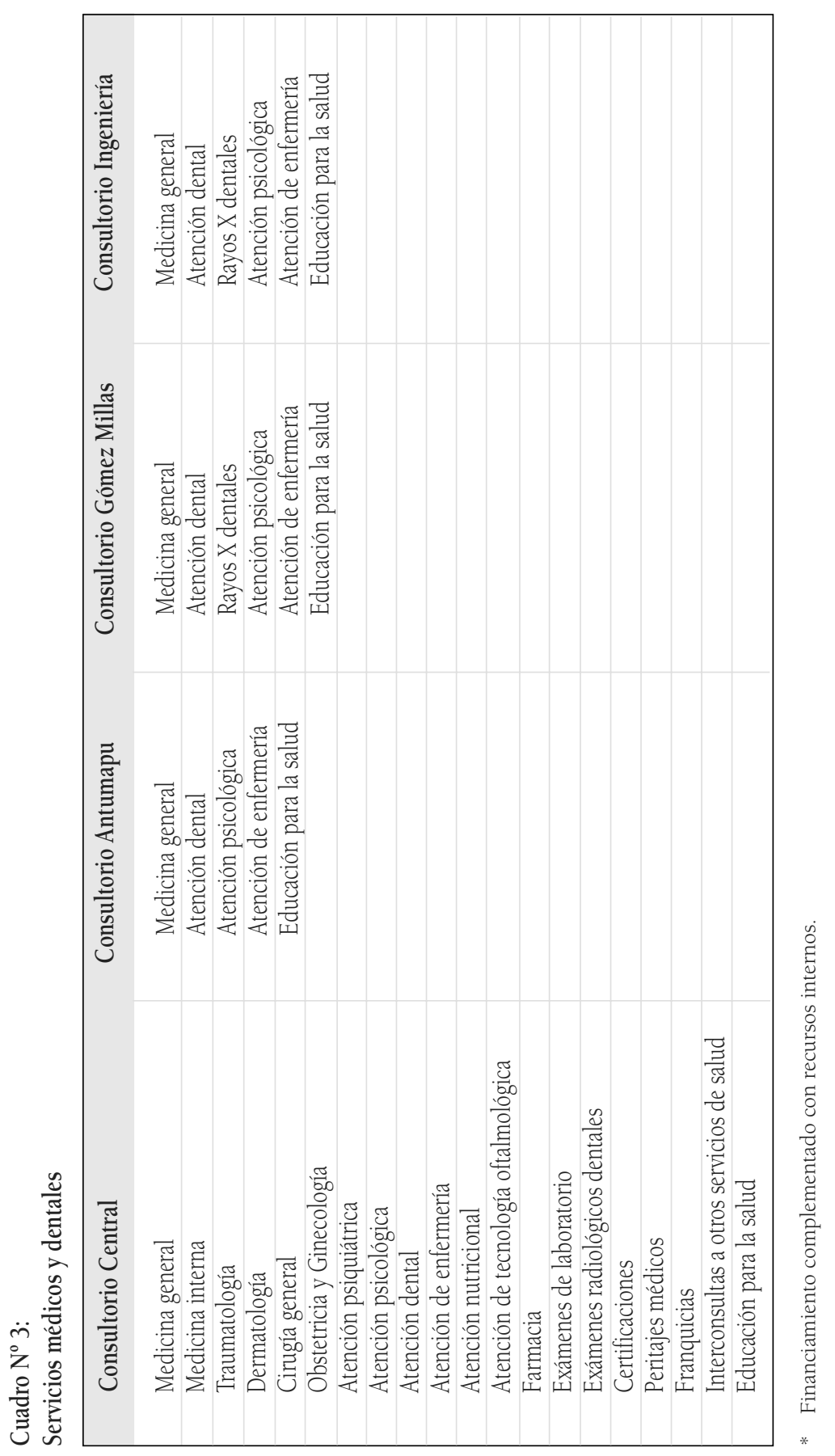




\section{APOYO A LAS ACTIVIDADES EXTRACURRICULARES}

La Universidad debe proveer "... programas y acciones que conduzcan a mejorar la calidad de vida de los estudiantes durante su permanencia en la institución, tanto para el apoyo del aprendizaje, como de actividades extracurriculares complementarias" (culturales, deportivas y recreativas, entre otras)*.

De acuerdo con sus orientaciones, la Universidad intenta contribuir a la promoción de acciones que busquen el mejoramiento de los proyectos estudiantiles con un enfoque transversal y colaborativo. En este ámbito, se entrega asesoría directa de acuerdo a los siguientes ámbitos de gestión:

- Evaluación de proyectos: Tiene por finalidad implementar una metodología que permita definir la viabilidad de apoyar iniciativas, teniendo en consideración aspectos como los resultados que se espera obtener, los mecanismos de emprendimiento, la replicabilidad de las propuestas y su sustentabilidad;

- Económica: Se apoyan los proyectos, dando esencialmente un apoyo técnico-económico, formulando las respectivas modificaciones, atendiendo al sentido de la iniciativa y considerando los criterios de empresa social;

- Legal: En esta área se inician los trámites que permitan la obtención del respectivo certificado de donaciones; junto con ello, y una vez concluido el proceso completo, se inicia una estrategia de sensibilización de la Ley de Donaciones en el mundo empresarial para apoyar iniciativas estudiantiles;

- Multimedios: Colaborará con los proyectos, generando el respectivo apoyo tecnológico en la presentación multimedial y soporte computacional, cuando se requiera;

- Institucional: Lleva a cabo las acciones pertinentes para desarrollar vinculaciones con organismos intra y extrauniversitarios, en la perspectiva de diseñar sistemas de fomento a la creatividad estudiantil y de favorecer el intercambio regional, nacional e internacional.

* Universidad de Chile, Orientaciones Estratégicas, Santiago, Chile, 2000-2005. 
La Universidad de Chile cuenta con un fondo concursable de proyectos al cual pueden acceder todos sus estudiantes. Este fondo lleva el nombre de Premio Azul, que financia total o parcialmente iniciativas voluntarias de los estudiantes en las áreas de extensión artística, comunicaciones, deportes, trabajo comunitario, infraestructura y encuentros temáticos en campus universitarios.

Más del 33\% de los proyectos han sido generados por alumnos en grupos interdisciplinarios. Uno de los objetivos del Premio Azul es precisamente estimular la comunicación transversal del estudiantado, a través de la generación de proyectos interfacultades, interdisciplinarios e interprofesionales, que ayuden a crear un cuerpo común tendiente a la percepción global de esta casa de estudios. Existe una enorme cantidad de proyectos e iniciativas que postulan al Premio Azul, lo que manifiesta la gran inquietud estudiantil por expresar las diversas formas de la creatividad y el compromiso con su Universidad y con el país. Entre estos destacan:

- Mejoramiento de espacios y relaciones comunitarias en el estamento estudiantil o en sectores postergados de nuestra sociedad;

- promoción de relaciones entre distintos colectivos estudiantiles que apunten a mejorar la calidad de vida;

- implementación de vínculos entre los estudiantes y las comunas adyacentes al campus universitario;

- trabajos voluntarios poblacionales;

- talleres dirigidos a sectores de escasos recursos;

- creación o continuidad de páginas web u otros sistemas multimediales digitalizados;

- entrega de servicios comunicacionales y coordinación entre los estudiantes a través de redes electrónicas;

- realización de ciclos culturales, festivales artísticos, exposiciones, consolidación de espacios y equipamientos para la extensión artística;

- difusión y convergencia pública de creaciones e iniciativas artísticas realizadas por los estudiantes;

- realización de competencias deportivas interfacultades e intercampus;

- realizaciones de encuentros de intercambio, diálogo, conversación y reflexión, así como asistencia u organización de congresos. Este conjunto queda enmarcado en un enfoque que pretende asistir a la articulación y participación transversal de estudiantes de distintas carreras, reflejado en postulaciones que contemplan responsables de más de una organización y una facultad. 
Gráfico 1: Proyectos extracurriculares apoyados 1999 / 2001

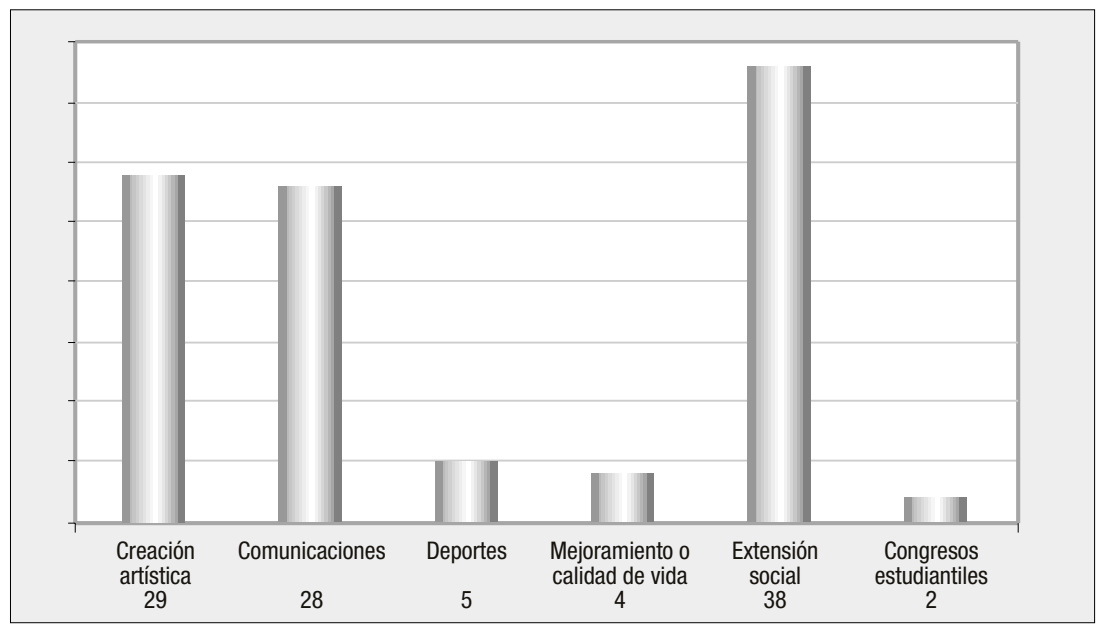

\section{CONSIDERACIONES FINALES}

La decisión de crear un sistema de beneficios estudiantiles, a comienzos del siglo XX, permitió desarrollar mecanismos que favorecieron el ingreso a y la mantención en la Universidad de estudiantes de escasos recursos, internalizando la necesidad de integrar a vastos sectores de la ciudadanía al desarrollo del país.

Actualmente, la Universidad de Chile desarrolla acciones tendientes a evolucionar desde una atención benéfico-asistencialista hacia un sistema público basado en los derechos sociales de los ciudadanos, con especial énfasis en los estudiantes más desfavorecidos o que enfrentan desiguales condiciones socioeconómicas para hacer frente a la vida universitaria. Así pues, nuestro eje central se sustenta en atender aquellos derechos humanos que interpelan directa o subsidiariamente a la institución, de tal forma que la Universidad se reconoce obligada a buscar las soluciones necesarias para que esos derechos sean ejercidos por todos en igualdad de oportunidades o, al menos, desde el mínimo que pide la equidad en la educación. 
Universidad de Chile, Orientaciones Estratégicas para la Reestructuración de Bienestar Estudiantil 2002.

Universidad de Chile, Dirección de Asuntos Estudiantiles, Informe de Gestión 1998-1999.

Universidad de Chile, Lineamientos Estratégicos de los Asuntos Corporativos en el Ámbito Estudiantil. 2001.

Universidad de Chile, Orientaciones Estratégicas de la Universidad de Chile 2000 - 2005.

Universidad de Chile, Políticas de la Dirección de Asuntos Estudiantiles 2001.

Universidad de Chile, Programa Actividades Estudiantiles 2002. 